\title{
Imagen corporal y práctica de actividades físico-deportivas en estudiantes de nivel secundaria
}

\section{The body image and the practice of physical-sport activities in middle school students}

\section{Imagem corporal e prática de atividades físico-desportivas em alunos do ensino secundário}

Ceballos-Gurrola, O.1, Medina-Rodríguez, R. E. 1, Juvera-Portilla, J. L. 1, Peche-Alejandro, P. 1, Aguirre-

López, L. F. 1, Rodríguez-Rodríguez, J. 1

1 Universidad Autónoma de Nuevo León

\section{RESUMEN}

El objetivo del estudio es valorar la imagen corporal, la práctica de actividades físico-deportivas y la percepción de la salud en escolares adolescentes de educación secundaria. Se presenta un diseño de estudio de tipo no experimental, descriptivo, comparativo y correlacional. La población objeto de estudio es una muestra de 416 estudiantes de educación secundaria de la ciudad de San Nicolás de los Garza, N. L. México seleccionados mediante un muestreo aleatorio y estratificado por género y grado escolar, con una edad entre 12 y 15 años $(13,14$ $\pm 0,87$ ), de los cuales 216 son hombres y 200 mujeres, con un peso promedio de 53,63 $\pm 10,33 \mathrm{~kg}$, una estatura de $1,59 \pm 8,44 \mathrm{~m}$ y un IMC de $21 \pm 3,03$. Se utilizó el cuestionario Body Shape Questionnaire (BSQ-8A), con 8 ítems y una escala de respuesta de 1 a 6 (donde 1 es nunca y 6 siempre), agrupados en puntos de corte sobre la procupación de la imagen corporal: < 19 ninguna, 19-25 leve, 26-33 moderada, > 33 marcada. El instrumento cuenta con buenas propiedades psicométricas. Para el análisis de los datos se utilizó el programa estadístico SPSS v24 y LISREL. En general los estudiantes adolescentes mexicanos tienen una preocupación importante de su imagen corporal, especialmente en las mujeres y los que perciben una mala salud, y esta no es determinante de la práctica de actividades físico-deportivas.

Palabras Clave: Imagen corporal, actividad física, salud, adolescentes.

\begin{abstract}
The aim of the study is to value body image, the practice of physical-sport activities, and the perception of the health in school age adolescents in middle school. A non-experimental, descriptive, comparative and correlational study design is presented. The subject population of the study is a sample of 416 middle school students from the city San Nicolas de los Garza, N. L. Mexico selected by random sampling and stratified by gender and grade level, with an age between 12 and 15 years $(13,14 \pm 0,87)$, of which 216 are male and 200 are female; with an average
\end{abstract}




\section{Imagen corporal y actividad físico-deportiva en jóvenes}

weight of 53,63 $\pm 10,33 \mathrm{~kg}$, a height of $1,59 \pm 8,44 \mathrm{~m}$ and a BMI of $21 \pm 3,03$. The questionnaire Body Shape Questionnaire (BSQ-8A) was used, with 8 items and a response scale of 1 to 6 (where 1 is never and 6 is always), grouped in cut off points based on the concern of the body image: < 19 never, 19-25 slight, 26-33 moderate, >33 marked. The instrument has good psychometric properties. The statistical program SPSS v24 and LISREL were used for the analysis of the data. In general, Mexican adolescent students have an important concern for their body image, especially in women and those who perceive poor health, people who have a higher practice of physicalsports activities show a positive attitude towards their own body.

Keywords: Body image, physical activity, health, adolescents

\section{RESUMO}

O objetivo do estudo é avaliar a imagem corporal, a prática de atividades físico-desportivas e a percepção de saúde em alunos adolescentes do ensino médio. Apresenta-se um desenho de estudo não experimental, descritivo, comparativo e correlacional. A população alvo é uma amostra de 416 estudantes do ensino médio da cidade de San Nicolás de los Garza, N. L. México, selecionado por amostragem aleatória e estratificado por gênero e série escolar, com idade entre 12 e 15 anos $(13,14 \pm 0,87)$, dos quais 216 são homens e 200 mulheres, com peso médio de 53,63 $\pm 10,33 \mathrm{~kg}$, altura de 1,59 $\pm 8,44 \mathrm{~m}$ e IMC de $21 \pm 3,03$. Foi utilizado o Body Shape Questionnaire (BSQ$8 \mathrm{~A}$ ), com 8 itens e uma escala de resposta de 1 a 6 (onde 1 nunca é e 6 sempre), agrupados em pontos de corte na preocupação com a imagem corporal: < 19 nenhum, 19-25 leve, 26-33 moderado, > 33 marcado. O instrumento tem boas propriedades psicométricas. O programa estatístico SPSS v24 e LISREL foram utilizados para a análise dos dados. Em geral, os estudantes adolescentes mexicanos têm uma preocupação importante com a sua imagem corporal, especialmente nas mulheres e naqueles que percebem a falta de saúde, e isso não é determinante para a prática de atividades físico-desportivas.

Palavras chave: Imagem corporal, atividade física, saúde, adolescentes.

\section{INTRODUCCIÓN}

Se ha comprobado en los últimos años, que en los países desarrollados se está produciendo un aumento de la obesidad e inactividad física en la etapa de la infancia y la adolescencia (Aranceta-Bartrina y PérezRodrigo, 2018; Guthold, Stevents, Riley, y Bull, 2018) y exponencialmente en los países que se encuentran en proceso de desarrollo (Álvarez-Dongo, SánchezAbanto, Gómez-Guizado, y Tarqui-Mamani, 2012). Este hecho se relaciona con los datos de la Organización Mundial de la Salud (OMS) en donde se considera que de los diez factores de riesgo identificados como claves para el desarrollo de enfermedades crónicas, cinco están estrechamente relacionados con la alimentación y el ejercicio físico (OMS, 2018). En consecuencia, la preocupación por la imagen corporal está presente en la edad escolar, y puede desembocar, en situaciones que provoquen trastornos de la conducta alimentaria y en un problema de salud pública. En este sentido, el presente trabajo se centra en analizar la imagen corporal, la práctica de actividades físico-deportivas y la percepción de la salud en escolares adolescentes de educación secundaria.

Se entiende la imagen corporal como un constructo teórico, que se estructura mediante varios factores, tales como vivencias de tipo afectivo y/o experiencias que van determinando y explicando algunos aspectos importantes de la personalidad, la autoestima o ciertas psicopatologías, tales como los trastornos de conducta alimentaria (Urrutia, Azpillaga, de Cos, y Muños, 2010); más recientemente, Piran (2016) define la imagen corporal como una construcción multifacética que se refiere a las actitudes y percepciones sobre el cuerpo, en particular su apariencia, y es una construcción más amplia que reconoce la importancia de las experiencias vividas por el cuerpo y la forma en que éste se relaciona con el mundo.

Entre los factores que influyen en este proceso durante el periodo de preadolescencia y adolescencia, son de importancia las relaciones que el alumnado desarrolla con su cuerpo, ya que estas van a repercutir en su autoestima, en la perspectiva sobre su imagen corporal 


\section{Apellidos, Autor (1), Apelidos, Autor (2),......}

y en las influencias de su grupo de pares (Schmidt, Valkanover, Roebers, y Conzelmann, 2013). La preadolescencia es una etapa del desarrollo humano donde influye más el aspecto social que el físico (Morgan, Saunders, y Lubans, 2012).

El estudiante tiene poco poder de decisión, en cambio es consumista; y eso le lleva a que determinados gustos e intereses se incentiven por una conveniencia del mercado y los medios de comunicación. También, suelen manifestar afinidad hacia una determinada estética impuesta por la sociedad, esto hace que los más jóvenes presenten una preocupación excesiva por su cuerpo y lleven a cabo conductas de riesgo que pueden producir trastornos de la conducta alimentaria, como anorexia y bulimia nerviosa (Baile, Raich, y Garrido, 2003; Xu et al., 2010), y además, hayan transcendido al mundo de la salud, tanto física, como mental (Kriemler et al., 2011); y durante la adolescencia se convierta en un problema de salud pública (Käll, Nilsson, y Lindén, 2014).

Por otro lado, autores señalan que existe una relación entre la percepción del estado de salud con la práctica físico-deportiva y la imagen corporal en adolescentes (Urrutia et al., 2010). La adolescencia es una etapa particularmente vulnerable en el desarrollo de la obesidad porque está marcada por una desaceleración del crecimiento y una disminución en los niveles de actividad física. Dado que existe un riesgo muy alto de sobrepeso, los adolescentes son propensos a ser adultos con sobrepeso, la participación de los jóvenes en la actividad física sigue siendo el objetivo esencial para la prevención de la obesidad (Stankov, Olds, y Cargo 2012) y sobretodo mantenimiento a largo plazo de la pérdida de peso (Veloigne et al., 2011), reuniendo todos estos beneficios para la salud y obtener un estilo de vida activo (Duncan et al., 2008).

En la mayoría de las teorías y modelos usados en el comportamiento y la investigación en ciencias sociales sobre la actividad física, la imagen corporal no se incluye directamente como una influencia de la construcción psicosocial integral (Glanz, Rimer, y Viswanath, 2008); sin embargo, la literatura ha indicado relaciones positivas entre la imagen corporal y la actividad física en adolescentes (Campbell y Hausenblas, 2009); así como en la práctica de otras disciplinas físico-deportivas como la danza (RequenaPérez, Martín-Cuadrado y Lago-Marín, 2015), usuarios de gimnasios de musculación (Baile-Ayensa, González-Díaz, Ramírez-Ortíz, y Suárez-Andujo,
2011), incluso en déficit de atención e hiperactividad de escolares (López-Sánchez, López-Sánchez, y DíazSuárez, 2015).

Por lo antes expuesto se plantea como objetivo valorar la imagen corporal, la práctica de actividades físicodeportivas y la percepción de la salud en escolares adolescentes de educación secundaria.

\section{MATERIAL Y MÉTODOS}

Se presenta un estudio comparativo, no experimental y transversal que analiza la relación entre la práctica de actividades físico-deportivas y la imagen corporal en estudiantes de educación secundaria (Ato, López, y Benavente, 2013).

\section{Participantes}

La población objeto de estudio son estudiantes de educación secundaria pública del sistema federal de la ciudad de San Nicolás de los Garza, Nuevo León, México $(n=16,849)$. Participó una muestra de 416 escolares, con un error del $\pm 5 \%$, seleccionados mediante un muestreo aleatorio y estratificado por género y grado escolar, con una edad entre 12 y 15 años $(13.14 \pm 0.87)$ de los cuales 216 son hombres y 200 mujeres, con un peso promedio de $53.63 \pm 10.33$ $\mathrm{kg}$, una estatura de $1.59 \pm 8.44 \mathrm{~m}$ y un IMC de $21 \pm$ 3.03 .

\section{Instrumentos}

Encuesta de Educación Física para la Salud (NúñezAvilés, 2015), contiene un apartado de datos generales (edad, estatura, peso y género), de la pregunta 1 a la 7 se refieren a la práctica de actividad físico-deportiva y percepción de la salud, de la 8 a la 15 se refieren a la imagen corporal "Body Shape Questionnaire" (Evans y Dolan, 1993-BSQ-8a) con 8 ítems que se puntuan con seis opciones de respuesta: (1) nunca, (2) raramente, (3) alguna vez, (4) a menudo, (5) muy a menudo y (6) siempre; agrupados en puntos de corte sobre la procupación de la imagen corporal: < 19 ninguna, 19-25 leve, 26-33 moderada, > 33 marcada. El instrumento cuenta con buenas propiedades psicométricas de validez y fiablilidad para esta población $($ Alfa $=0.89 ; \mathrm{KMO}=0.90 ; g l=28, \mathrm{p}<0.01$; $57.30 \%$ de la varianza total). Los índices de ajuste y error son adecuados $\chi 2 / g l=2.92 ; \mathrm{CFI}=0.97 ; \mathrm{NNFI}=$ $0.96 ;$ RMSEA $=0.9$. 


\section{Imagen corporal y actividad físico-deportiva en jóvenes}

\section{Procedimiento}

Para su aplicación, se solicitó el permiso previo a realizar el estudio a las autoridades de las escuelas secundarias, los cuestionarios se administraron en un salón de clase por una persona capacitada donde se explicó brevemente el objetivo del estudio y aclaración de posibles dudas. Se contó con el consentimiento informado por parte de los padres de familia.

\section{Análisis estadístico}

Para el análisis de los datos, se utilizaron los programas estadísticos SPSS v24 y LISREL 8,80 (Jöreskog y Sörbom, 2006). Se obtuvieron datos descriptivos y pruebas de normalidad mediante Kolmogorov-Smirnov, asimetría y curtosis; así como la comparación de los puntos de corte de la preocupación de la imagen corporal mediante tablas de contingencia por sexo, salud y práctica de actividad físico-deportiva (chi cuadrada). Para la validez y fiabilidad se obtuvo el alfa de Cronbach y se realizó un análisis factorial exploratorio (AFE), utilizando la medida de adecuación muestral KMO y prueba de Tabla 1.

Estadísticos descriptivos y prueba de normalidad de los items esfericidad de Bartlett; mediante el método de extracción de componentes principales, y con el empleo de un criterio de rotación oblicua (Varimax). Posteriormente se realizó el análisis factorial confirmatorio (AFC) atendiendo la naturaleza ordinal de las variables. Se consideraron los criterios de coeficiente $\chi 2 / \mathrm{gl}$ inferior a 3,0 para considerarse un buen ajuste del modelo (Kline, 2005). Los índices de CFI y NNFI por encima de 0,90 que indican un ajuste aceptable (Hu y Bentler, 1999). Para la RMSEA, se consideran valores satisfactorios menores a 0,05; y aceptables, valores inferiores a 0,08 (Llorent-Segura, Ferreres-Traver, Hernández-Baeza, y Tomás-Marco, 2014).

\section{RESULTADOS}

Se presentan los resultados sobre la imagen corporal y práctica de actividades físico-deportivas en estudiantes de nivel secundaria. La Tabla 1, muestra los estadísticos descriptivos y se afirma que no existe una normalidad para los ítems según los valores de asimetría, curstosis y Kolmogorov-Smirnov ( $\mathrm{p}<$ $0,01)$.

\begin{tabular}{ccccccc}
\hline Items & $\mathrm{N}$ & Media & DT & Asimetría & Curtosis & $K-S$ \\
\hline Item 1 & 416 & 2.86 & 1.39 & 55 & -.36 & .000 \\
Item 2 & 416 & 2.37 & 1.30 & .69 & -.30 & .000 \\
Item 3 & 416 & 1.99 & 1.28 & 1.25 & .82 & .000 \\
Item 4 & 416 & 1.91 & 1.25 & 1.27 & .72 & .000 \\
Item 5 & 416 & 2.37 & 1.41 & .98 & .21 & .000 \\
Item 6 & 416 & 2.28 & 1.41 & .99 & .12 & .000 \\
Item 7 & 416 & 2.63 & 1.56 & .67 & -.61 & .000 \\
Item 8 & 416 & 3.33 & 1.75 & .19 & -1.23 & .000 \\
\hline
\end{tabular}

Nota: $K-S=$ Prueba de normalidad Kolmogorov-Smirnov

En cuanto a la preocupación de la imagen corporal, se encuentran diferencias significativas entre el grupo de los hombres y las mujeres, siendo estos últimos

Tabla 2.

Nivel de preocupación de la imagen corporal según sexo los que tienen los valores con mayor preocupación (Tabla 2; $\mathrm{p}<0.01$ ).

\begin{tabular}{ccccc}
\hline Nivel de & Hombre & Mujer & Total & P \\
preocupación & $n(\%)$ & $n(\%)$ & $n(\%)$ & valor \\
\hline
\end{tabular}




\section{Apellidos, Autor (1), Apelidos, Autor (2),......}

\begin{tabular}{lcccc}
\hline Ninguna & $149(69 \%)$ & $75(37.5 \%)$ & $224(53.8 \%)$ & \\
Leve & $46(21.3 \%)$ & $42(21.0 \%)$ & $88(21.2 \%)$ & $\mathbf{. 0 0 0}$ \\
Moderada & $16(7.4 \%)$ & $52(26.0 \%)$ & $68(6.3 \%)$ & \\
Marcada & $5(2.3 \%)$ & $31(5.5 \%)$ & $36(8.7 \%)$ & \\
Total & $216(51.9 \%)$ & $200(48.1 \%)$ & $416(100 \%)$ & \\
\hline
\end{tabular}

El $14,9 \%$ de los estudiantes declara nunca practicar actividades físico-deportivas y muestran cierta preocupación por su imagen corporal, el $8,7 \%$ lo hace en vacaciones, el $33,2 \%$ de vez en cuando, el $10,8 \%$ en fines de semana y el 32,5\% frecuentemente. Aunque se observa que varían los momentos de práctica, ésta no muestra diferenias significativas según el nivel de preocupación de la imagen corporal (Tabla $3 ; \mathrm{p}>0,05$ ).

Tabla 3.

Nivel de preocupación de la imagen corporal de acuerdo a la práctica de actividades físico-deportivas

\begin{tabular}{llllllll}
\hline \multicolumn{1}{c}{$\begin{array}{c}\text { Nivel de } \\
\text { preocupación }\end{array}$} & Nunca & Vacaciones & $\begin{array}{c}\text { De vez en } \\
\text { cuando }\end{array}$ & $\begin{array}{c}\text { Fines de } \\
\text { semana }\end{array}$ & Frecuentemente & Total & $\begin{array}{c}\text { P } \\
\text { valor }\end{array}$ \\
\hline Ninguna & $34(54.8 \%)$ & $22(61.1 \%)$ & $67(48.6 \%)$ & $21(46.7 \%)$ & $80(59.3 \%)$ & $224(53.8 \%)$ & \\
Leve & $7(11.3 \%)$ & $7(19.4 \%)$ & $34(24.6 \%)$ & $11(24.4 \%)$ & $29(21.5 \%)$ & $88(21.2 \%)$ & $\mathbf{. 2 2 0}$ \\
Moderada & $17(27.4 \%)$ & $5(13.9 \%)$ & $23(16.7 \%)$ & $9(20.0 \%)$ & $14(10.4 \%)$ & $68(16.3 \%)$ & \\
Marcada & $4(6.5 \%)$ & $2(5.6 \%)$ & $14(10.1 \%)$ & $4(8.9 \%)$ & $12(8.9 \%)$ & $36(8.7 \%)$ & \\
Total & $62(14.9 \%)$ & $36(8.7 \%)$ & $138(33.2 \%)$ & $45(10.8 \%)$ & $135(32.5 \%)$ & $416(100 \%)$ & \\
\hline
\end{tabular}

La preocupación de la imagen corporal y la percepción de la salud es considerada como positiva, por lo que se supone que ambas tiene un incremento de acuerdo a su percepción (Tabla 4; $\mathrm{p}>0.05$ )

Tabla 4

Nivel de preocupación de la imagen corporal de acuerdo a la percepción de la salud

\begin{tabular}{lccccc}
\hline \multicolumn{1}{c}{$\begin{array}{c}\text { Nivel de } \\
\text { preocupación }\end{array}$} & Buena & Regular & Mala & Total & $\begin{array}{c}\text { P } \\
\text { valor }\end{array}$ \\
\hline Ninguna & $147(55.9 \%)$ & $74(51.4 \%)$ & $3(33.3 \%)$ & $224(53.8 \%)$ & \\
Leve & $55(20.9 \%)$ & $30(20.8 \%)$ & $3(33.3 \%)$ & $88(21.2 \%)$ & \\
Moderada & $36(13.7 \%)$ & $29(20.1 \%)$ & $3(33.3 \%)$ & $68(16.3 \%)$ & $\mathbf{. 3 3 2}$ \\
Marcada & $25(9.5 \%)$ & $11(7.6 \%)$ & $0(0.0 \%)$ & $36(8.7 \%)$ & \\
Total & $263(63.2 \%)$ & $144(34.6 \%)$ & $9(2.2 \%)$ & $416(100 \%)$ & \\
\hline
\end{tabular}

\section{DISCUSIÓN}

La imagen corporal es considerada como una representación mental amplia de la figura corporal, su forma y tamaño, la cual está influenciada por diversos factores (Slade, 1994). Para su medición, se utilizan principalmente cuestionarios validados de imagen corporal (Blanco, Solano, Benavides, y Ornelas, 2017), uno de los más referenciados es el "Body Shape
Questionnaire" cuenta con varias versiones, el original con 34 ítems, además de 16, 14 y 8, este último instrumento con cuatro versiones: A, B, C y D permitiendo elegir el que más se ajusta al contexto (da Silva, Dias, Maroco, y Campos, 2014; Pook, TuschenCaffier y Brähler, 2008; Welch, Lagerström, y Ghaderi, 2012). En este trabajo su utilizó el BSQ-8A 


\section{Imagen corporal y actividad físico-deportiva en jóvenes}

(Evans y Dolan, 1993), se confirma que cuenta con buenas propiedades psicométricas de validez $\mathrm{y}$ fiablilidad para la población de escolares de educación secundaria de México, se obtiene un solo factor mediante el AFE y adecuados índices de ajuste y error con el AFC.

Los factores que son comúnmente asociados a la imagen corporal son el estatus socioeconómico, peso corporal, edad y el sexo (Latiff, Muhamad, y Rahman, 2018); en este estudio se encontró que las mujeres tienen una marcada preocupación con relación a los hombres, esto puede ser debido a una mayor insatisfacción corporal por las mujeres que generalmente han tenido una mayor preocupación por su estética (Damiano et al., 2015; Guedea-Delgado, Solano-Pinto, Blanco-Ornelas, Ceballos-Gurrola, y Zueck-Enríquez, 2017; Heron, Smyth, Akano, y Wonderlich, 2013; Latiff et al., 2018; Ramos, Rivera, Pérez, Lara, y Moreno, 2016).

La práctica de actividades físico-deportivas está asociada con la imagen corporal; es decir las personas que tienen una mayor práctica muestran una actitud más positiva hacia su propio cuerpo (Campbell y Hausenblas, 2009; Kantanista, Osiński, Borowiec, Tomczak, y Król-Zielińska, 2015; Williams y Cash, 2001). Resalta la importancia de esta información, ya que se ha comprobado que la imagen corporal se la relaciona también con el estado de salud y la autoestima (Urrutia et al., 2010; Vaquero, Alacid, Muyor, y López-Miñarro, 2013). Los datos del presente trabajo coinciden con los resultados señalados, ya que independientemente del momento de práctica de actividad física, ya sea en vacaciones, fines de semana o de manera frecuente, disminuye la preocupación por la imagen corporal. Por otro lado, el deporte de élite puede relacionarse negativamente con la preocupación de la imagen corporal ocasionando trastornos de conducta alimentaria e influencia sociocultural de la delgadez (Williamson et al., 1995), especialmente en deportes como la gimnasia rítmica, patinaje artístico o deportes de resistencia (Moreira, Macêdo, y Fontes, 2018; Vaquero et al., 2013).

Algunos autores (Käll et al., 2014; Oliva-Peña et al., 2016; Urrutia et al., 2010) describen la relación que se da entre la percepción del estado de salud y la imagen corporal percibida es significativa y positiva, por lo que se supone que a mejor percepción de la imagen corporal mejor percepción de estado de salud y viceversa, coincidiendo con los resultados obtenidos en esta investigación. Las consecuencias de la insatisfacción con la imagen corporal son variadas, así cursa con pobre autoestima, depresión, ansiedad social, inhibición y disfunciones sexuales, desarrollo de trastornos de la conducta alimentaria, quirúrgicos, dermatológicos, capilares, entre otros, de forma reiterada y con demandas irracionales (Hollander, Cohen, y Simeon, 1993).

\section{CONCLUSIONES}

Se puede concluir que en general los estudiantes adolescentes mexicanos tienen una preocupación importante de su imagen corporal, especialmente en las mujeres y los que perciben una mala salud, las personas que tienen una mayor práctica de actividades físico-deportivas muestran una actitud más positiva hacia su propio cuerpo. Como futuras líneas de investigación sería conveniente analizar la imagen corporal en estudiantes adolescentes diferenciando las disciplinas deportivas que practican con regularidad y los que no tienen el hábito.

\section{APLICACIONES PRÁCTICAS}

Los resultados de este estudio aportan información de interés hacia los entrenadores, instructores o docentes, sobre la percepción que tienen los adolescentes sobre su imagen corporal a la hora de diseñar, desarrollar e implantar programas de actividad físico-deportiva destinados a la prevención de enfermedades y desarrollo de estilos de vida activos y saludables. Así mismo, las diferencias de género encontradas, apuntan a la necesidad de tener en cuenta las distintas percepciones de mujeres y hombres para aumentar las probabilidades de éxito de las intervenciones. Resulta ser una buena alternativa el uso del cuestionario de imagen corporal en su versión corta BSQ-8A en adolescentes mexicanos, ya que se obtuvieron buenas propiedades psicométricas de validez y fiablilidad.

\section{REFERENCIAS}

1. Álvarez-Dongo, D., Sánchez-Abanto, J., GómezGuizado, G. y Tarqui-Mamani, C. (2012). Sobrepeso y obesidad: prevalencia y determinantes sociales del exceso de peso en la población Peruana (2009-2010). Rev Peru Med Exp Salud Pública, 29(3), 303-313. 


\section{Apellidos, Autor (1), Apelidos, Autor (2),......}

2. Aranceta-Bartrina, J. y Pérez-Rodrigo, C. (2018). La obesidad infantil: una asignatura pendiente. Rev Esp Cardiol, 71(11), 888-891. https://doi.org/10.1016/j.recesp.2018.04.038

3. Ato, M., López, J. J. y Benavente, A. (2013). Un sistema de clasificación de los diseños de investigación en psicología. Anales de Psicología, 29(3), 1038-1059.

4. Baile-Ayensa, J. I., González-Díaz, A., RamírezOrtíz, C. y Suárez-Andujo, P. (2011). Imagen corporal, hábitos alimentarios y hábitos de ejercicio físico en hombres usuarios de gimnasios y hombres universitarios no usuarios. Revista de Psicología del Deporte, 20(2), 353-366.

5. Baile, J. I., Raich, R. M. y Garrido, E. (2003). Evaluación de la insatisfacción corporal en adolescentes: efecto de administración de una escala. Anales de psicología, 19(2), 187-192.

6. Blanco, J. R., Solano, N., Benavides, E. V. y Ornelas, M. (2017). Composición e invarianza factorial del cuestionario IMAGEN en adolescentes mexicanos y españoles. Cuadernos de Psicología del Deporte, 17(2), 35-44.

7. Campbell, A. y Hausenblas, H. A. (2009). Effects of exercise interventions on body image: Ametaanalysis. Journal of Health Psychology, 14, 780793. org/10.1177/1359105309338977.

8. da Silva, W. R., Días, J. C., Maroco, J. y Campos, J. (2014). Confirmatory factor analysis of different versions of the Body Shape Questionnaire applied to Brazilian university students. Body Image, 11(4), 384-390. http://dx.doi.org/10.1016/j.bodyim.2014.06.001

9. Damiano, S. R., Gregg, K. J., Spiel, E. C., Mclean, S.A., Wertheinm, E. H. y Paxton, S. J. (2013). Relationships between body size attitudes and body image of 4 year old boys and girls, and attitudes of their father and mothers. J Eat Disord, 3(1): 16. doi: 10.1186/s40337-015-0048-0

10. Duncan, G., Goldberg, J., Noonan, C., Velnez, A., Hurvitz, P. y Buchwald, D. (2008). Unique environmental effects on physical activity participation: a twin study. PLoS One, 3(4), e2019.

https://doi.org/10.1371/journal.pone.0002019
11. Evans, C. y Dolan, B. (1993). Body Shape Questionnaire: derivation of shortened alternate forms. International Journal of Eating Disorders, 13(3), 315-321.

12. Glanz, K., Rimer, B. K. y Viswanath, K. (2008). Health behavior and health education: Theory, research, and practice. San Francisco: JosseyBass.

13. Guedea-Delgado, J. C., Solano-Pinto, N., BlancoOrnelas, J. R., Ceballos-Gurrola, O. y ZueckEnríquez, M. C. (2017). Autoconcepto físico, género y cuidado de la salud en universitarios mexicanos. Revista de Psicología del Deporte, 26(2), 51-59.

14. Guthold, R., Stevents, G. A., Riley, L. M. y Bull, F. (2018). Worldwide trends in insufficient physical activity from 2001 to 2016: a pooled analysis of 358 population-based surveys with 1.9 million participants. Lancet Glob Health, 6, e1077-86. http://dx.doi.org/10.1016/S2214109X(18)30357-7

15. Heron, K. E., Smyth, J. M., Akano, S. A. y Wonderlich, E. H. (2013). Assessing body imagen in young children: a preliminary study of racial and developmental differences. SAGE Open; 3(1), 1-7. https://doi.org/10.1177/2158244013478013

16. Hollander, E., Cohen, L. J. y Simeon, D. (1993). Body dysmorphic disorder. Psychiatric Annals, 23(7), 359-364. https://doi.org/10.3928/00485713-19930701-06

17. Hu, L. y Bentler, P. M. (1999). Cutoff criteria for fit indexes in covariance structure analysis. Conventional criteria versus new alternatives. Structural Equation Modeling, 6, 1-55. doi:10.1080/10705519909540118

18. Jöreskog, K. y Sörbom, D. (2006). LISREL 8.80. Structural equation modeling with the simplis command language (Software de cómputo). Chicago, EU: Scientific Software International.

19. Käll, L. B., Nilsson, M. y Lindén, T. (2014). The impact of a physical activity intervention program on academic achievement in a Swedish elementary school setting. Journal of school health, 84(8), 473-480. https://doi.org/10.1111/josh.12179 


\section{Imagen corporal y actividad físico-deportiva en jóvenes}

20. Kantanista, A., Osiński, W., Borowiec, J., Tomczak, M., Król-Zielińska, M. (2015). Body image, BMI, and physical activity in girls and boys aged 14-16 years. Body image, 15, 40-3. Doi:10.1016/j.bodyim.2015.05.001

21. Kline, R. B. (2005). Principles and practice of structural equationmodeling. New York, NY: The Guilford Press.

22. Kriemler, S., Meyer, U., Martin, E., van Sluijs, E., Andersen, L. y Martin, B. (2011). Effect of school-based interventions on physical activity and fitness in children and adolescents: a review of reviews and systematic update. British Journal of Sports Medicine, 45(11), 923-30. doi: 10.1136/bjsports-2011-090186

23. Latiff, A., Muhamad, M. y Rahman, R. (2018). Body image dissatisfaction and its determinnants among young primary-school adolescents. Journal of Taibah University Medical Science, 13(1), 34-41. http://dx.doi.org/10.1016/j.jtumed.2017.07.003

24. Lloret-Segura, S.; Ferreres-Traver, A.; Hernández-Baeza, A. y Tomás-Marco, I. (2014). El análisis factorial exploratorio de los ítems: una guía práctica, revisada y actualizada. Anales de psicología, 30(3), 1151-1169. https://doi.org/10.6018/analesps.30.3.199361

25. López-Sánchez, L., López-Sánchez, G. F. y DíazSuárez, A. (2015). Efectos de un programa de actividad física en la imagen corporal de escolares con TDAH. Cuadernos de Psicología del Deporte, 15(2), 135-142.

26. Moreira, M. L., Macêdo, A. A. y Fontes, S. (2018). Satisfaçâo da imagem corporal em jovens praticantes de ginastica em grupo. Revista Brasileira de Obesidade, Nutriçâo e Emagrecimento, 12(71), 400-405.

27. Morgan, P. J., Saunders, K. L. y Lubans, D. R. (2012). Improving physical self-perception in adolescent boys from disadvantaged schools: psychological outcomes from the physical activity leaders randomized controlled trial. PediatrObes, 7, e27-e32. Doi:10.1111/j.20476310.2012.00050.x

28. Nuñez-Avilés, F. (2015). Estudio de la satisfacción de la imagen corporal en educación primaria. Relaciones con la actividad física y el nivel de condición física de los escolares. Tesis de Doctorado, Universidad de Málaga, Málaga, España.

29. Oliva-Peña, Y., Ordoñez-Luna, M., SantanaCarvajal, A., Marín-Cárdenas, A., Andueza, G. y Gómez, I. (2016). Concordancia del IMC y la percepción de la imagen corporal en adolescentes de una localidad suburbana de Yucatán. Revista Biomédica, 27, 49-60. http://revistabiomedica.mx/index.php/revbiomed /article/view/24/28.

30. Organización Mundial de la Salud. (2018). Nota informativa 23 de febrero. Actividad Física. Recuperado de http://www.who.int/mediacentre/factsheets/fs38 5/es/.

31. Piran, N. (2016). Embodied possibilities and disruptions: The emergence of the experience of embodiment construct from qualitative studies with girls and women. Body Image, 18, 43-60. http://dx.doi.org/10.1016/j.bodyim.2016.04.007

32. Pook, M., Tuschen-Caffier, B. y Brähler, E. (2008). Evaluation and comparison of different versions of the Body Shape Questionnaire. Psychiatry Research, 158(1), 67-73. http://dx.doi.org/10.1016/j.psychres.2006.08.002

33. Ramos, P., Rivera, F., Pérez, R. S., Lara, L. y Moreno, C. (2016). Diferencias de género en la imagen corporal y su importancia en el control de peso. Escritos de Psicología, 9(1), 42-50. doi: 10.5231/psy.writ.2015.1409

34. Requena-Pérez, C. M., Martín-Cuadrado, A. M. y Lago-Marín, B. S. (2015). Imagen corporal, autoestima, motivación y rendimiento en practicantes de danza. Revista de Psicología del Deporte, 24(1), 37-44.

35. Schmidt, M., Valkanover, S., Roebers, C. y Conzelmann, A. (2013). Promoting a functional physical self-concept in physical education: Evaluation of a 10-week intervention. European Physical Education Review, 19(2), 232-255. https://doi.org/10.1177/1356336X13486057

36. Slade, P. D. (1994). ¿What is body image? Behaviour Research and Therapy, 32, 497-502. https://doi.org/10.1016/0005-7967(94)90136-8 


\section{Apellidos, Autor (1), Apelidos, Autor (2),......}

37. Stankov, I., Olds, T. y Cargo, M. (2012). Overweight and obese adolescents: what turns them off physical activity. International journal of behavioral nutrition and physical activity, 9, 53. https://doi.org/10.1186/1479-5868-9-53

38. Urrutia, S., Azpillaga, I., de Cos, G. L. y Muñoz, D. (2010). Relación entre la percepción de estado de salud con la práctica físico-deportiva y la imagen corporal en adolescentes. Cuadernos de Psicología del Deporte, 10(2), 51-56.

39. Vaquero, R., Alacid, F., Muyor, J. M. y LópezMiñarro, P. A. (2013). Imagen corporal; revisión bibliográfica. Nutr Hosp, 28(1), 27-35. http://dx.doi.org/10.3305/nh.2013.28.1.6016

40. Veloigne, M., Bourdeaudhuij, I., Tanghe, A., D'Hondt, E., Theuwis, L., Vansteenkiste, M. y Deforche, B. (2011). Self-determined motivation towards physical activity in adolescents treated for obesity: an observational study. International Journal of Behavioral Nutrition and Physical Activity, 8, 97. https://doi.org/10.1186/14795868-8-97

41. Welch, E., Lagerström, M. y Ghaderi, A. (2012). Body Shape Questionnaire: Psychometric properties of the short version (BSQ-8C) and norms from the general Swedish population. Body Image, 9(4), 547-550. doi: 10.1016/j.bodyim.2012.04.009

42. Williams, P. A. y Cash, T. F. (2001). Effect of a circuit weight training program on the body images of collage students. Int $\mathbf{J}$ Eat Disord, 30(1), 75-82. https://doi.org/10.1002/eat.1056

43. Williamson, D. A., Netemeyer, R. G., Jackman, L. P., Anderson, D. A., Funsch, C. L. y Rabalais, J. Y. (1995). Structural equation modeling of risk-factors for the development of eating disorder symptoms in female atheletes. Int $\mathbf{J}$ Eat Disord, 17(4), https://doi.org/10.1002/1098$108 X(199505) 17: 4<387::$ AIDEAT2260170411>3.0.CO;2-M

44. Xu, X., Mellor, D., Kiehne, M., Ricciardelli, L. A., McCabe, M. P., y Xu, Y. (2010). Body dissatisfaction, engagement in body change behaviors and sociocultural influences on body image among Chinese adolescents. Body image, $7(2)$, 156-164. https://doi.org/10.1016/j.bodyim.2009.11.003 
Imagen corporal y actividad físico-deportiva en jóvenes 